Production scientifique des CHU-CHR en 2006-2015 : évolutions et positionnement national

Publication output of french university hospitals during 2006-2015: trends and position in the national health research landscape

Baudoin Lesya ${ }^{1}$

Akiki Vincent ${ }^{1}$

Magnan Antoine ${ }^{1,2}$

Devos Patrick ${ }^{1,3}$

1 - CNCR, 52 rue Maurice Ripoche, 75014 Paris, France

2 - CHU de Nantes, Nantes, France

3 - Univ. Lille, CHU Lille, EA 2694 - Santé publique : épidémiologie et qualité des soins, F59000 Lille, France

Correspondance : Baudoin Lesya, CNCR, 52 rue Maurice Ripoche, 75014 Paris

Email : lesya.baudoin@cncr.fr

Remerciements : les auteurs remercient tous les relecteurs de ce manuscrit qui ont contribué à son amélioration.

\title{
Liens d'intérêts :
}

L. B., V. A. et P.D. : salariés au Groupement de coopération sanitaire Comité National de Coordination de la Recherche (CNCR).

A. M. : Président du GCS CNCR. 


\section{Résumé}

Objectif

Identifier la production scientifique des CHU dans la base de données internationale Web of Science et produire les indicateurs bibliométriques comparatifs permettant de la positionner parmi les acteurs de recherche biomédicale en France.

\section{Méthodes}

Afin de disposer de données fiables, la première étape a consisté à unifier toutes les variantes d'adresses des 32 CHU/CHR dans le Web of Science sous la forme d'Organization-Enhanced. Les indicateurs bibliométriques (nombre de publications, indice de citation normalisé, Top $1 \%$ Top $10 \%$, etc.) ont été calculés pour analyser la production des CHU et des organismes membres d'Aviesan.

\section{Résultats}

En termes des volumes de production, les CHU sont le $3^{\text {e }}$ producteur national des publications, après les universités et le CNRS; ils contribuent à environ $17 \%$ de la production française et à 2,5\% de la production européenne (UE28). Au cours des 10 ans, l'impact de ces publications augmente plus vite que le volume. La visibilité et l'impact de publications des CHU reflètent leurs activités de recherche, d'enseignement et de transfert.

\section{Conclusion}

Cette étude a permis d'objectiver la production scientifique des CHU et de démontrer la place majeure qu'ils occupent dans la recherche biomédicale française, en étant à l'interface de la recherche translationnelle et du patient. 


\section{English Summary}

Objectives

The aim of the current study is to identify the output of French university hospitals in the Web of Science database and to perform a comparative bibliometric analysis to characterize their position in the national biomedical research landscape.

Methods

The first stage consists in creation of the unified "Organization-Enhanced" records in the Web of Science for 32 university hospitals, in order to ensure the reliable aggregated data. The research output of university hospitals and organizations members of the French National Alliance for Life Sciences and Health (Aviesan) are analyzed using bibliometric indicators, including the number of publications, category normalized citation impact, top one percent and top 10 percent of the most cited papers, etc.

Results

In terms of publication output university hospitals rank 3rd in France, after universities and CNRS. They contribute to about $17 \%$ of the total national production and to $2,5 \%$ of the European publications (EU28). Over the 10 years period, the impact of the papers produced by university hospitals grows faster than volume. Visibility and impact comply with the university hospitals' core assignments: teaching, research and knowledge transfer.

\section{Conclusion}

This study provides objective data showing a leading position of the university hospitals among the biomedical research organizations in France, playing a major role in patient-based research. 
Ce qui était connu

- Selon les données SIGAPS, les CHU contribuent à $90 \%$ de la production scientifique des établissements de santé.

- Au cours de ces 10 années, les CHU ont augmenté quantitativement (nombre de publications) et qualitativement (pourcentage d'articles classés A ou B) leur production scientifique.

- Le nombre et la proportion de publications en langue française des CHU ont fortement baissé au profit des publications en anglais, ces dernières représentant désormais plus de $80 \%$ des publications.

Ce qu'apporte l'article

- L'impact d'une procédure d'unification des adresses sur la visibilité des CHU

- Des données en termes de positionnement des CHU par rapport aux autres acteurs de la recherche biomédicale

- Une analyse des collaborations des CHU

- Des indicateurs d'impact permettant de mesurer la portée scientifique de la recherche menée dans les CHU 


\section{Introduction}

Les analyses statistiques de la production scientifique des opérateurs de recherche sont largement utilisées comme outils d'aide à l'évaluation et au pilotage de l'activité de recherche. Les données source, longtemps limitées à l'unique base Science Citation Index, se diversifient et démocratisent, ainsi que leur utilisation en bibliométrie, autrefois réservée aux initiés. Grâce à la profusion des publications sur internet, via les bases payantes ou gratuites, les archives ouvertes ou les revues électroniques, la recherche des documents est devenue rapide et aisée. Les données de citations, en plus des bases commerciales Web of Science (WoS) et Scopus, sont accessibles gratuitement via des indexes comme Google Scholar ou des initiatives telles que Open Citations. Toutefois, malgré l'accessibilité et l'abondance des ressources, l'agrégation des données de publications au niveau institutionnel reste difficile, entravée par l'absence de normalisation dans les noms des organismes. En France, la situation se complique par la présence des structures mixtes formant le fameux «millefeuille », les tutelles multiples s'efforçant chacune de revendiquer la production réalisée. Le recensement de la production institutionnelle reste un problème majeur en bibliométrie, sa mise en œuvre étant très consommatrice en temps et en ressources et la qualité pas toujours garantie.

Le Système d'Interrogation, de Gestion et d'Analyse des Publications Scientifiques (SIGAPS) développé par le CHU de Lille adopte une approche différente pour identifier la production : elle est basée sur les noms des auteurs affiliés à l'établissement. L'homonymie résultante d'une interrogation nominative de la base PubMed est résolue ensuite par la validation individuelle des auteurs. Outre son utilisation dans le cadre de la répartition des financements des Missions d'enseignement, de recherche, de référence et d'innovation (MERRI), SIGAPS qui est déployé dans l'ensemble des établissements de santé, est employé comme un outil de pilotage de recherche par les hôpitaux, mais aussi comme une base pour les analyses bibliométriques [1,2]. Cependant, SIGAPS étant limité aux établissements de santé, leur 
production, bien qu'identifiée, ne peut être positionnée vis-à-vis d'autres acteurs au niveau national ou international.

Dans le cadre de ses missions de promotion et de valorisation de la recherche réalisée dans les Etablissements Publics de Santé, le Comité National de Coordination de la Recherche (CNCR) s'est investi dans un ambitieux projet visant à rendre visible la production des Centres Hospitaliers Régionaux et Universitaires (CHU) dans le Web of Science, base de référence historique pour la bibliométrie. Ce travail a abouti à un rapport d'analyse de la production des CHU publié par le CNCR [3]. Le présent article met l'accent sur la méthodologie de repérage de la production employée et expose les principaux résultats de cette analyse.

\section{Méthodes}

\section{Corpus}

L'analyse s'appuie sur les données de la base internationale et multidisciplinaire Web of Science (WoS), Core Collection éditée par la société Clarivate Analytics. Les indicateurs ont été calculés à partir des données consolidées dans la plateforme InCites, outil analytique du même éditeur permettant d'agréger les statistiques de production et de citation aux niveaux des pays, organismes et disciplines. Le jeu de données utilisées correspond à la mise à jour de la base WoS du 9 Septembre 2017. Pour les années 2006-2015, seuls les articles originaux (documents de type «Articles » dans le WoS) ont été pris en compte.

\section{Indicateurs}

\section{$\underline{\text { Indicateur de production }}$}

Nombre de publications associé à un acteur, identifié à l'aide des affiliations. Il est calculé ici en compte de présence : une publication est attribuée à un acteur (organisme, pays...) dès lors qu'au moins un chercheur de cette entité figure sur la liste des co-auteurs, quelle que soit sa 
position. Chaque publication est donc comptabilisée autant de fois qu'il y a d'acteurs (organismes, pays...). Ce type de comptage ne permet pas l'addition des données (somme $>100 \%$ ). Le compte de présence traduit la participation de l'acteur à l'activité de publication. Indice de citation normalisé (ICN):

Cet indice est calculé comme le nombre de citations reçues par article et normalisé par rapport à la moyenne mondiale de citation des articles de la même discipline, de même année de publication et pour le même type de document. La valeur de l'indice montre si les publications de l'acteur sont en moyenne plus $(>1)$ ou moins $(<1)$ citées que celles de la référence (Monde).

Top $10 \%$ et Top $1 \%$ de citation :

Ces indicateurs mesurent la part (en \%) d'articles d'un acteur appartenant au $10 \%$ et au $1 \%$ d'articles les plus cités au niveau international. Chaque article est considéré dans le contexte de son champ disciplinaire, le premier décile (10\%) et le centile (1\%) sont définis dans le périmètre des fréquences de citation propres à chaque discipline, en fonction de l'année de publication et du type de document.

$\underline{\text { Part de publications (\%) en langue anglaise ou en langue française }}$

Cet indice est calculé par rapport à la production globale d'un acteur, il montre dans quelle mesure la production d'un acteur cible le public national ou international.

$\underline{\text { Part de production }(\%) \text { en collaboration internationale : }}$

Cet indice est calculé comme le pourcentage de publications avec au moins deux pays cosignataires.

\section{Choix de références}

L'analyse porte sur trente-deux établissements : 30 CHU (27 CHU métropolitains et 3 CHU d'outre-mer) et 2 CHR, constituant le périmètre des « CHU». Leur production est mise en regard avec celle de la France et de l'ensemble des pays de l'Union européenne (UE28). A 
titre de comparaison institutionnelle, nous présentons les indicateurs des établissements membres d'Aviesan en les complétant avec un établissement de soins, UNICANCER, membre associé d'Aviesan.

\section{Unification des adresses dans WoS}

Afin de pallier à l'hétérogénéité des noms d'établissements dans le WoS, Clarivate Analytics a mis en place une fonctionnalité permettant de rassembler toutes les dénominations d'un établissement existantes dans la base et de les unifier sous un nom unique (Organization Enhanced).

Les corpus des membres d'Aviesan ont été constitués sur la base des «Organizations Enhanced » respectifs, pour la même période et le même type de document. Ce mode de repérage présente l'avantage de la reproductibilité et de la transparence des résultats obtenus, dans la mesure où l'index des « Organizations Enhanced » est accessible à tous les utilisateurs du WoS et d'InCites. Toutefois, la fiabilité de l'unification n'est pas la même pour tous les établissements. Plus particulièrement, l'unification des affiliations des universités dans WoS a été réalisée de façon hétérogène : certaines universités ont intégré l'ensemble des publications des hôpitaux appartenant à leurs CHU, d'autres se sont limitées aux adresses où l'université est mentionnée explicitement.

Bien qu'il ne paraisse pas pertinent de sélectionner ce groupe d'organismes aux périmètres instables comme comparateur de référence, il est impossible en même temps de faire l'impasse sur le secteur universitaire compte tenu de son rôle majeur. Pour cette raison, les indicateurs des universités sont présentés dans ce rapport à titre indicatif. 


\section{Résultats}

\section{Repérage des publications des CHU et unification des adresses dans WoS}

L'objectif de ce travail était d'identifier, pour chaque $\mathrm{CHU}$, le maximum de variantes existantes dans la base WOS afin d'en définir des règles fiables. La procédure employée est représentée schématiquement sur la Figure 1.

SIGAPS dispose des corpus validés de publications des CHU référencées dans PubMed, depuis 2000. Nous avons extrait de la base nationale SIGAPS les PMID (PubMed IDentifier) des publications des 32 CHU parues entre 2000 et 2014. Les fonds documentaires des bases PubMed et WoS ne sont pas identiques ; aussi, afin d'ajuster le périmètre du corpus issu de PubMed à celui du WoS, nous avons pris en compte uniquement les publications dans les revues des catégories SIGAPS A-E (soit les revues ayant un facteur d'impact et présentes dans le WoS). Les identifiants des publications correspondantes à ces critères ont été transmis à la société Clarivate Analytics, qui a réalisé l'appariement dans sa base et repéré les identifiants WoS (clés UT) correspondants. Sur 317114 publications PubMed, 279850 ont été ainsi identifiées dans la base WoS.

A notre demande, l'ensemble des adresses correspondantes à ces publications nous a été fourni ensuite par Clarivate Analytics. Pour chacun des $32 \mathrm{CHU}$, nous avons sélectionné les adresses faisant référence à l'établissement en tenant compte d'au minimum 2 éléments : un élément qui désigne l'établissement et un élément qui indique la position géographique (ville, pays) de l'hôpital. Les listes de variantes ainsi constituées ont été ensuite revues et validées par les correspondants des établissements. Adressées à l'équipe d'unification de Clarivate Analytics, elles ont servi à construire des règles qui définissent 1' «Organization Enhanced » de chacun des établissements. Chaque association « organisme - lieu » retenue comme règle identifie un établissement d'une manière non ambiguë. Le nombre de variantes identifiées par établissement varie entre 100 et 5000, en fonction de la taille du CHU. 
Pour contrôler la qualité de l'unification, nous avons comparé, pour chaque CHU, le volume de publications associées à son «Organization Enhanced» avec celui identifié dans SIGAPS, pour la même période et les mêmes types de documents. La mesure de rappel, couramment utilisée en science de l'information est calculée de façon suivante :

$$
\text { Rappel }=\frac{N b \text { de documents correctement attribués à l'Ets dans WoS }}{N b \text { de documents validés pour l'Ets dans SIGAPS * }}
$$

* sur le périmètre ajusté (hors les revues sans le facteur d'impact)

L'examen de l'état d'identification de la production des CHU dans WoS avant le démarrage des travaux avait mis en évidence les taux de rappel allant de $12 \%$ à $88 \%$ et la médiane de 52 $\%$. A l'issu du projet, nous avons observé les taux de rappel de $42 \%$ à $96 \%$, avec une médiane de $83 \%$.

\section{Production scientifique des CHU et d'autres membres d'Aviesan}

L'unification a permis d'identifier dans le WoS, sur la période 2006 à 2015, plus de 112000 articles avec au moins une adresse faisant référence à un CHU. Toutes disciplines confondues, les CHU-CHR contribuent à environ $17 \%$ de la production française et à $2,5 \%$ de la production européenne (UE28). Le Tableau I présente les volumes de production par membre d'Aviesan pour deux périodes consécutives, ainsi que leurs parts respectives dans la production française et européenne (UE28). Malgré la tendance générale à l'augmentation du nombre des publications, les parts respectives des organismes dans la production nationale et européenne restent relativement stables dans le temps.

La production des CHU se démarque parmi les membres d'Aviesan par sa concentration dans le domaine médical $^{1}$ : les publications dans ce champ constituent près de $90 \%$ du corpus des CHU. Les publications dans le domaine médical sont majoritaires dans la production de l'Inserm et d'UNICANCER (plus de 70\%), elles représentent près de la moitié de la

\footnotetext{
${ }^{1}$ MEDICAL AND HEALTH SCIENCES selon la classification OCDE [4]
} 
production de l'Institut Pasteur. En comparaison, les publications médicales sont minoritaires pour le CNRS, INRA et l'IRD (moins de $20 \%$ ) et pour le CEA ou l'Inria (moins de 10\%).

L'impact, mesuré par l'indice de citation normalisé, des publications des organismes présentés dans le Tableau II se situe au-dessus des moyennes européenne et française. Le CNRS maintient son niveau d'impact aux environs de 1,3 de manière constante (i. e. $\simeq 30 \%$ au-dessus de la moyenne mondiale), tandis que l'indice de l'Inserm se stabilise autour de 1,8. UNICANCER se démarque par le niveau très élevé d'impact de ses publications qui, de plus, progresse fortement au cours des 10 ans. L'impact des publications des CHU affiche une croissance rapide : proche de la moyenne mondiale en 2006, il atteint 1,7 ( $\simeq 70 \%$ au-dessus de la moyenne mondiale) en 2015, soit une augmentation de 57\%. Ce taux de croissance est en effet le plus important parmi les organismes présentés.

En ce qui concerne les classes de publications très citées, la position des CHU témoigne d'une dynamique positive : la part de production dans le Top 10\%, toutes disciplines confondues (Figure 2A), passe de 14\% en 2006-2010 à 16,9\% en 2011-2016. Sur cette figure, la diagonale représente une situation où la proportion de cette tranche des publications est stable entre deux périodes. Lorsque la dynamique de l'indicateur est orientée à la hausse, le centre du cercle se situe au-dessus de la diagonale ; inversement, une baisse proportionnelle de cette part d'articles positionne le centre du cercle en-dessous de la diagonale. Les tracées donnent des repères de référence pour l'UE28 et la France. La part d'articles au Top 10\% dans la production des $\mathrm{CHU}$, du CEA, d'UNICANCER et de l'Institut Pasteur est en forte progression.

La progression des CHU est encore plus marquante dans la tranche des publications citées au Top 1\% (Figure 2B). Globalement, plus d'un article français sur 4 de cette catégorie est cosigné par les auteurs des CHU. 
Les CHU publient proportionnellement plus en français que d'autres membres d'Aviesan : la part de publications en français dans leur production, bien que fortement diminuée en 10 ans, passant de $24 \%$ à 13\%, reste la plus élevée dans le panel, devant UNICANCER (Tableau III). Le CEA et l'Inria sont les instituts qui publient le moins en français.

Comparativement aux autres organismes, les CHU sont moins impliqués dans les collaborations internationales (Tableau IV). La part de publications internationales ne représente pas plus de 30\% du corpus des CHU en 2006-2010. Une forte progression de cette part entre les deux périodes ( $+8,5$ points) ne permet cependant pas de rattraper l'écart avec les organismes de recherche impliqués internationalement depuis longtemps.

Les CHU coopèrent à l'échelle nationale avec des nombreux acteurs publics et privés. Les partenariats noués au sein de l'Alliance pour les Sciences de la Vie et de la Santé jouent un rôle essentiel, les publications co-produites avec les établissements d'Aviesan sont les plus nombreuses dans le corpus des CHU. La coopération se concentre dans différentes disciplines, en fonction des profils spécifiques aux établissements partenaires. Le Tableau V met en évidence les 10 premières disciplines en commun avec chacun des membres d'Aviesan. La neurologie clinique et les neurosciences se trouvent en première ligne de la collaboration de plusieurs organismes. La biologie moléculaire, l'immunologie et la génétique, disciplines transversales pour les sciences du vivant, interviennent dans plusieurs partenariats. Notons des profils similaires de collaborations avec les Instituts Pasteur et l'IRD, focalisés sur la microbiologie, la virologie, et les maladies infectieuses, thématiques phares de ces instituts. Les collaborations avec l'INRA sont majoritairement tournées vers les domaines de la nutrition et du métabolisme, et des collaborations avec l'Inria vers les domaines de l'ingénierie et de l'informatique appliquée.

L'Inserm est le partenaire majeur de la recherche menée dans les CHU. Au fil des années la collaboration entre les CHU et l'Inserm ne cesse de croitre : le nombre d'articles en commun 
a plus que doublé en 10 ans (Figure 3). La croissance de la production commune est supérieure à la croissance de la production propre de chacun des deux partenaires.

La répartition des articles selon les disciplines définies par les revues de publication (WoS Categories) met en évidence les axes majeurs de la recherche menée au sein des CHU et de l'INSERM ainsi que les axes communs (Figure 4). Dans la partie basse du graphique se retrouvent les disciplines avec participation majoritaire des $\mathrm{CHU}$ : chirurgie, cardiologie et maladies cardiovasculaires, pédiatrie, urologie et néphrologie, maladies respiratoires, obstétrique et gynécologie, pour n'en citer que quelques-unes. A l'inverse, les disciplines plus présentes dans le corpus de l'Inserm sont positionnées plus près de l'axe de l'ordonnée. Sans surprise, il s'agit des disciplines plus fondamentales : biochimie et biologie moléculaire, biologie cellulaire, génétique, médecine expérimentale, etc. Les disciplines dans lesquelles la contribution de l'Inserm et des CHU est proportionnellement égale se positionnent autour de la diagonale du graphique. Parmi ces dernières figurent : oncologie, neurosciences, immunologie, pharmacologie, hématologie, maladies infectieuses, etc.

\section{Discussion}

Les analyses bibliométriques, ainsi que les classements internationaux, des institutions ayant une activité de recherche reposent sur leur repérage à partir des adresses des auteurs. La normalisation des noms des organismes revêt donc une importance grandissante dans ce contexte, et plus particulièrement en France, en raison de la complexité des tutelles des structures de recherche et la difficulté pour les chercheurs d'utiliser une adresse normalisée. L'étude de la problématique des affiliations réalisée par Dassa et al [5] présente un panorama d'outils visant à remédier aux lacunes constatées : chartes de signature édictées par les institutions, curation des données par les éditeurs des bases. Ces mesures restent, malheureusement, d'une portée limitée. Même si l'approche d'unification via 
l'«Organization Enhanced» proposée par Clarivate Analytics reste pas complétement satisfaisante (règles d'unification parfois approximatives et mises à jour chronophage des listes de variantes), elle a l'avantage d'être partagée et visible par tous les utilisateurs du WoS, ce qui permet de reproduire les résultats. L'unification réalisée ainsi pour les 32 CHU-CHR a permis d'identifier la production des établissements de façon satisfaisante, avec un rappel médian de $83 \%$ par rapport aux données SIGAPS qui peuvent être considérées comme un corpus de référence. Ce taux de rappel dépend essentiellement du respect ou non-respect d'une mention du CHU, sous une variante identifiée, dans les signatures des auteurs, les auteurs signant parfois avec leur adresse hospitalière, universitaire ou celle de leur équipe de recherche.

Il n'existe que très peu de publications permettant d'évaluer la fiabilité des «Organization Enhanced» des établissements du périmètre Aviesan. Une étude sur la production des membres d'Aviesan a été réalisée par l'Observatoire des Sciences et des Techniques (OST), sur les données de publications de l'année 2006 [6]. Cette étude montre que, pour la majorité des organismes, l'identification de la production via l' «Organization Enhanced » donne des résultats cohérents, l'exception faite du CNRS. Le charactère évolutif et une très grande mixité des structures, associés à une volumétrie très importante, ne facilitent pas l'unification des données d'affiliations de cet organisme. Il est donc probable que nos données sousestiment les indicateurs de production du CNRS. Quant aux universités, leurs « Organization Enhanced » sont d'une qualité variable, l'identification étant rendue difficile par des réformes structurelles successives, ainsi que par un manque d'homogénéité dans la définition de leurs périmètres.

Malgré les limites signalés, l'unification des données des CHU dans WoS a permis, pour la première fois, une analyse mettant en regard la production des $\mathrm{CHU}$ avec celles des membres 
de l'Alliance Nationale pour les Sciences de la Vie et de la Santé regroupant les acteurs institutionnels majeurs de la recherche biomédicale.

En termes des volumes de production et tous domaines confondus, les CHU sont le $3^{\mathrm{e}}$ producteur national, après les universités et le CNRS. Toutefois, il s'agit, pour chacun des acteurs, de travaux de recherche majoritairement collaboratifs, avec la participation souvent indissociable de plusieurs partenaires institutionnels. La progression du nombre de publications des $\mathrm{CHU}$ entre deux périodes est moindre que celle des organismes partenaires. Cet indicateur, dépendant de la taille, doit être mis en regard avec l'évolution des effectifs des chercheurs dans chacun de ces organismes. Malheureusement nous ne disposons pas de ces données.

L'impact des publications des CHU mesuré par l'indice de citation normalisé est supérieur à la référence française mais moindre que celui de l'Institut Pasteur, d'UNICANCER, de l'Inserm ou du CEA. Toutefois cet indice progresse plus rapidement dans le corpus des CHU que dans ceux d'autres membres d'Aviesan.

La part des publications présentes dans les Top $10 \%$ ou du Top $1 \%$ augmente de manière importante et plus d'un article français sur 4 cité au Top $1 \%$ est cosigné par un auteur d'un CHU.

Les publications en français sont relativement plus présentes dans la production des CHU par rapport aux autres organismes. Cette spécificité reflète l'importance des publications à caractère pédagogique ou de transfert destinées aux professionnels de santé. En accord avec les missions d'enseignement et des soins, cette dimension sous-tende toutefois la baisse des indicateurs d'impact des CHU en comparaison avec les organismes dont la production est presque exclusivement anglophone.

L'analyse des collaborations montre que les CHU sont pleinement intégrés dans l'écosystème national de la recherche. Le volume de publications avec l'Inserm, partenaire privilégié, a 
doublé en 10 ans. L'analyse par discipline met en évidence une complémentarité des compétences dans les disciplines fondamentales et cliniques, ainsi que les axes qui focalisent ces deux approches, comme l'oncologie, les neurosciences ou l'immunologie.

Le pourcentage de publications en collaboration internationale des $\mathrm{CHU}$ est plus faible que celui des autres membres d'Aviesan. Ces chiffres rejoignent le rapport produit par la National Science Foundation [7] qui montre que la médecine, les sciences de l'ingénieur et les sciences sociales sont, globalement, les domaines qui affichent les plus bas taux de collaboration internationale. Le développement des échanges internationaux présente donc un potentiel de visibilité important pour la recherche des CHU, les publications issues de collaborations internationales étant généralement plus citées que les autres [8-14].

\section{Conclusion}

Nous avons mis en place et validé une méthodologie d'unification des adresses dans le WoS en utilisant les données existantes issues de SIGAPS. L'approche utilisée a permis d'identifier la production des $\mathrm{CHU}$ avec un niveau de rappel satisfaisant, de façon reproductible, et d'augmenter sa visibilité.

Nous avons produit une série d'indicateurs comparatifs objectivant, pour la première fois, la place importante des CHU dans la recherche française. Au cours des 10 ans, la production des CHU a suivi une évolution positive, aussi bien du point de vue quantitative (volume) que qualitative (indicateurs d'impact et de visibilité). Les résultats de notre étude montrent une spécificité de la production des CHU qui réunit, parallèlement aux articles d'excellence très visibles, des publications d'une moindre visibilité et souvent en langue française, reflet de leur mission de formation à la recherche. La position des CHU dans le paysage national nous paraît stratégique : étroitement liés aux universités et aux EPST, ils jouent un rôle moteur 
dans la production des connaissances au bénéfice des patients, dans la dissémination de l'expertise et favorisent l'innovation dans les pratiques cliniques. 


\section{Références}

[1] Baudoin L, Devos P. Analyse bibliométrique des publications des CHU-CHR 2005-2014. Coordination Nationale des CHU en matière de recherche et d'innovation (CNCR); 2016. p. 39.

[2] Devos P, Cortot A, Ménard J. Utilisation des données SIGAPS pour mesurer l'impact du plan Alzheimer sur la production d'articles des CHU. La Presse Médicale. 2016;45:e1-e10.

[3] Production scientifique des CHU : positionnement et évolution 2006-2015. Comité National de Coordination de la Recherche; 2017. p. 39.

[4] Organisation for Economic Co-operation and Development. Revised field of science and technology (FOS) classification in the Frascati manual. Paris: Organisation for Economic Cooperation and Development (OECD); 2007.

[5] Dassa M, Deniau A, Durand-Barthez M, Girard F, Pothier N, Sene A. Le millefeuille des affiliations françaises dans les publications scientifiques. Documentaliste-Sciences de l'Information. 2014;51:12-6.

[6] Filliatreau G, Laville F, Ramanana-Rahary S, Taillibert M-L, Teixeira N, Vincent R. Indicateurs bibliométriques sur la production scientifique, en sciences de la vie, des institutions membres de l'Alliance nationale pour les sciences de la vie et de la santé (AVIESAN). Observatoire des Sciences et des Techniques; 2009. p. 29.

[7] National Science B. Science and Engineering Indicators 2016. Arlington, VA: National Science Foundation; 2016. p. 898.

[8] Katz JS, Hicks D. How much is a collaboration worth? A calibrated bibliometric model. Scientometrics. 1997;40:541-54.

[9] Van Raan AFJ. The influence of international collaboration on the impact of research results - Some simple mathematical considerations concerning the role of self-citations. Scientometrics. 1998;42:423-8. 
[10] Glanzel W. Coauthorship patterns and trends in the sciences (1980-1998): A bibliometric study with implications for database indexing and search strategies. Libr Trends. 2002;50:461-73.

[11] Franceschet M, Costantini A. The effect of scholar collaboration on impact and quality of academic papers. Journal of Informetrics. 2010;4:540-53.

[12] Bordons M, Aparicio J, Costas R. Heterogeneity of collaboration and its relationship with research impact in a biomedical field. Scientometrics. 2013;96:443-66.

[13] Didegah F, Thelwall M. Which factors help authors produce the highest impact research? Collaboration, journal and document properties. Journal of Informetrics. 2013;7:861-73.

[14] Smith MJ, Weinberger C, Bruna EM, Allesina S. The Scientific Impact of Nations: Journal Placement and Citation Performance. PLOS ONE. 2014;9:e109195. 


\section{Légendes des figures}

FIGURE 1 : Schéma de la procédure d'unification des adresses dans WoS.

FIGURE 2 : Evolution des parts des publications citées au niveau du Top 10\% (A) et du Top $1 \%$ (B) dans les corpus des membres d'AVIESAN et de références, toutes disciplines confondues, entre 2006-2010 et 2011-2015.

FIGURE 3 : Evolution du nombre de publications en collaboration entre les CHU et l'Inserm par rapport au nombre de publications hors de ce partenariat, 2006-2015.

FIGURE 4 : Vue d'ensemble des disciplines des publications issues de la collaboration CHUInserm. 


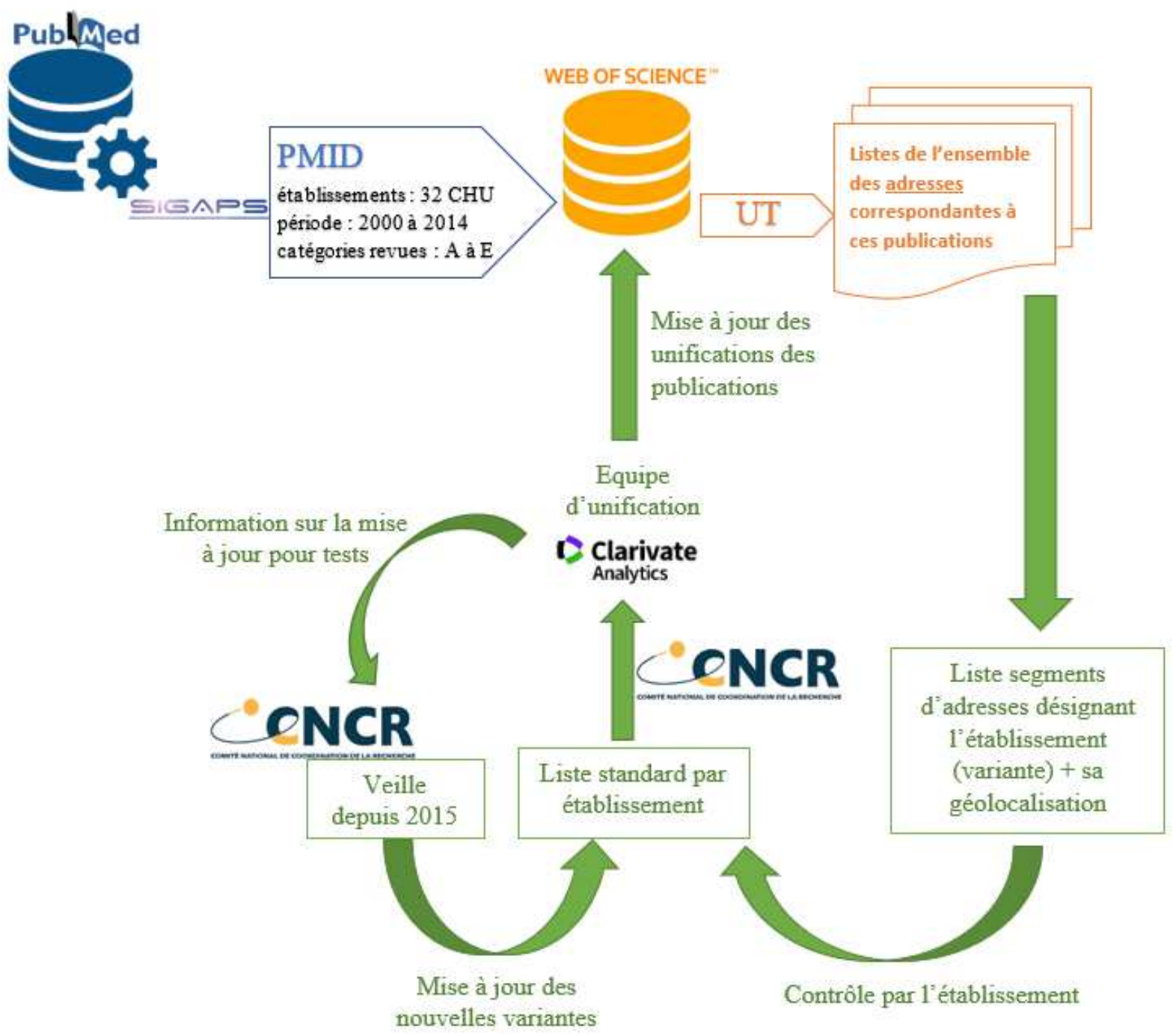

\section{FIGURE 1}

Schéma de la procédure d'unification des adresses dans WoS. 


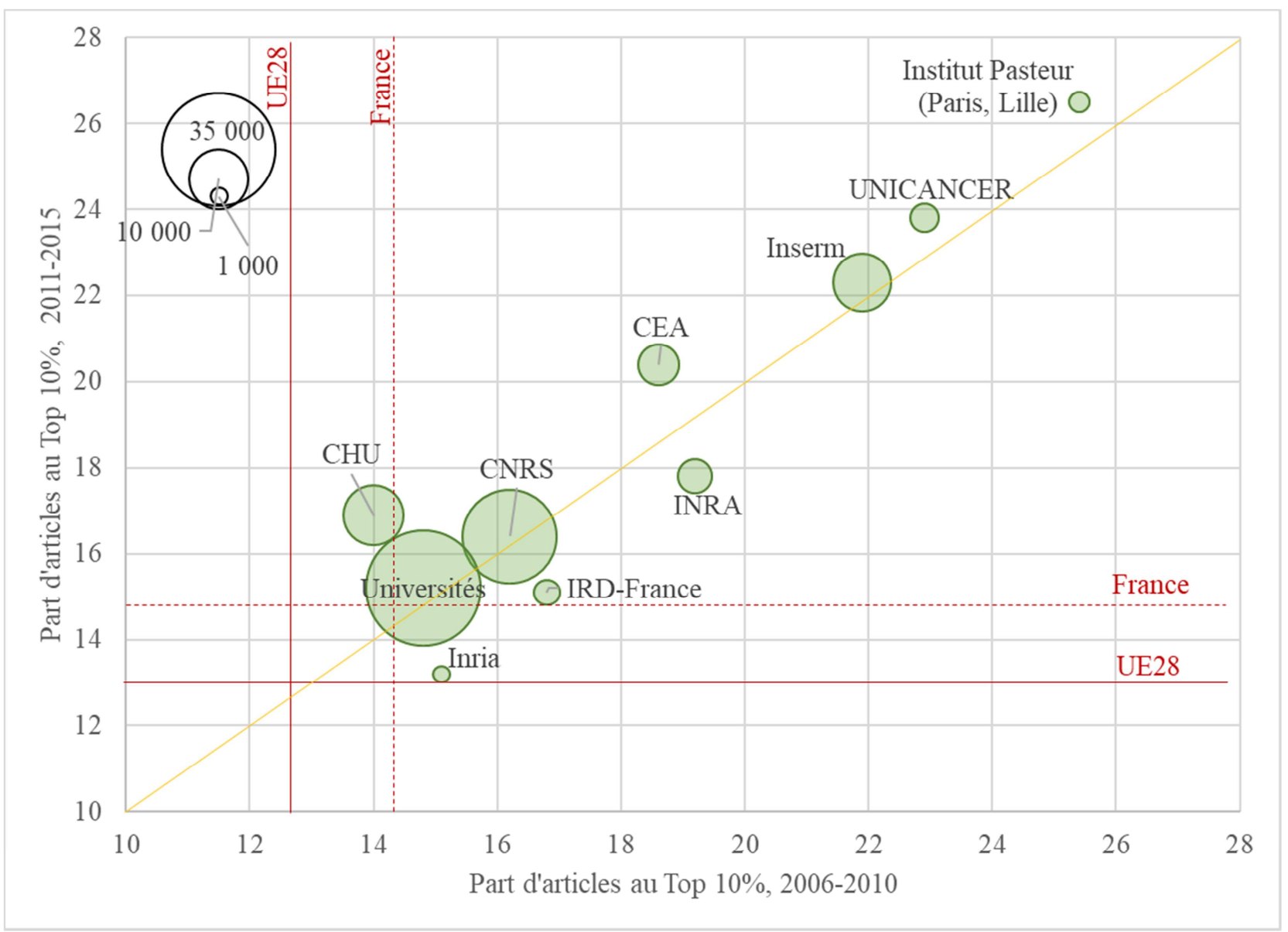

\section{FIGURE 2A}

Evolution des parts des publications citées au niveau du Top 10\% dans les corpus des membres d'AVIESAN et de références, toutes disciplines confondues, entre 2006-2010 et 2011-2015. 


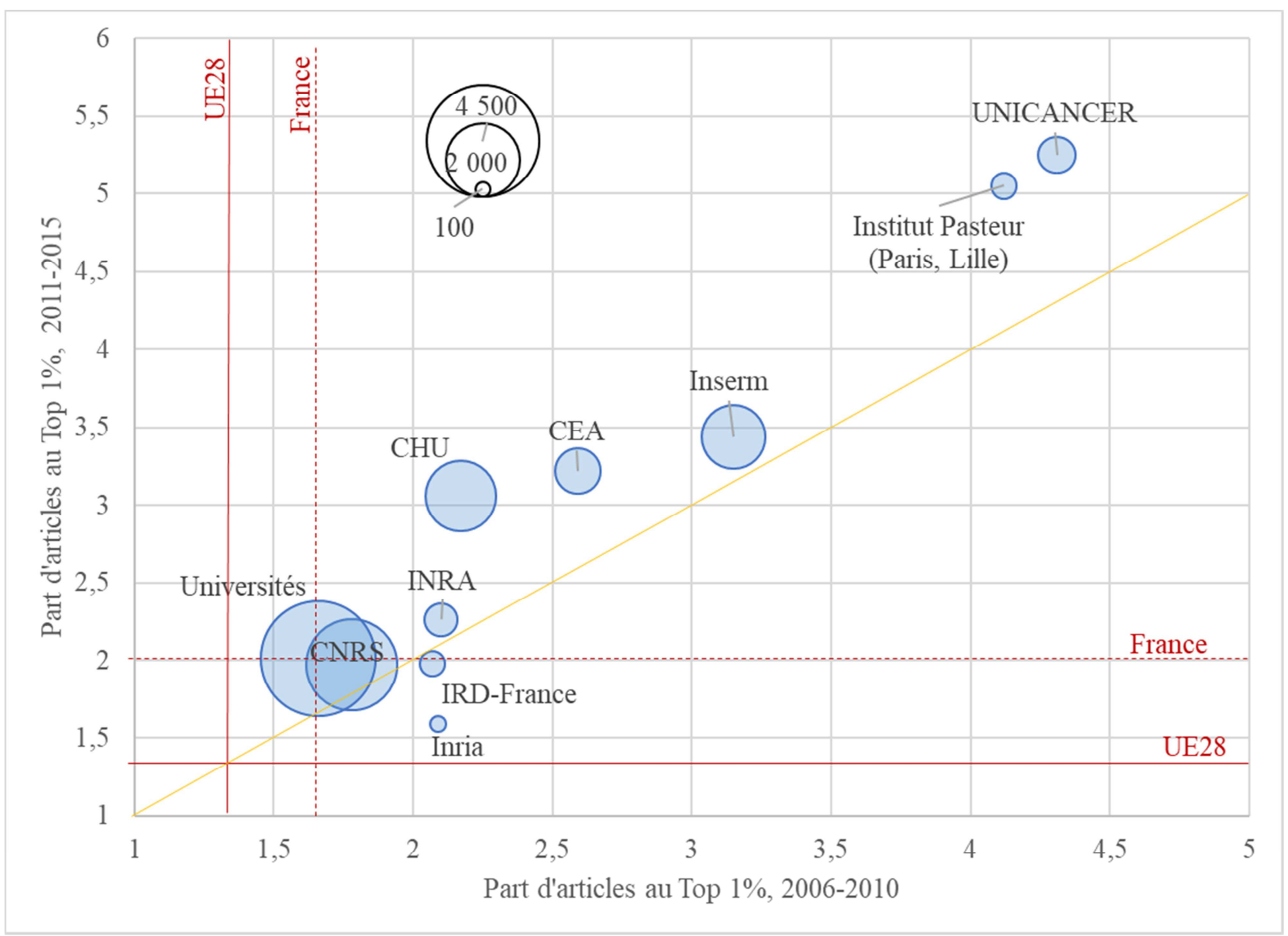

FIGURE 2B

Evolution des parts des publications citées au niveau du Top $1 \%$ dans les corpus des membres d'AVIESAN et de références, toutes disciplines confondues, entre 2006-2010 et 2011-2015. 


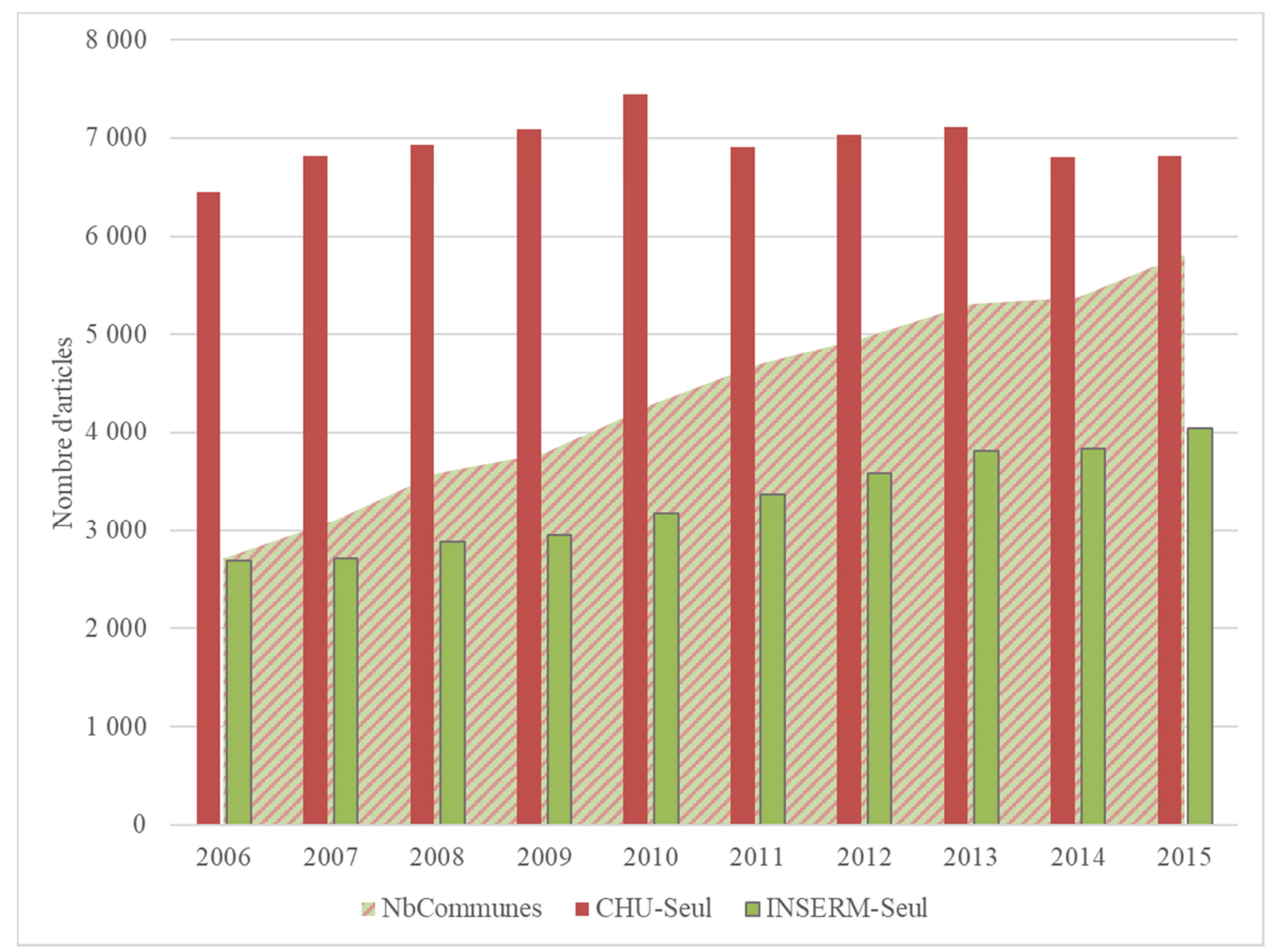

\section{FIGURE 3}

Evolution du nombre de publications en collaboration entre les CHU et l'Inserm par rapport au nombre de publications hors de ce partenariat, 2006-2015 


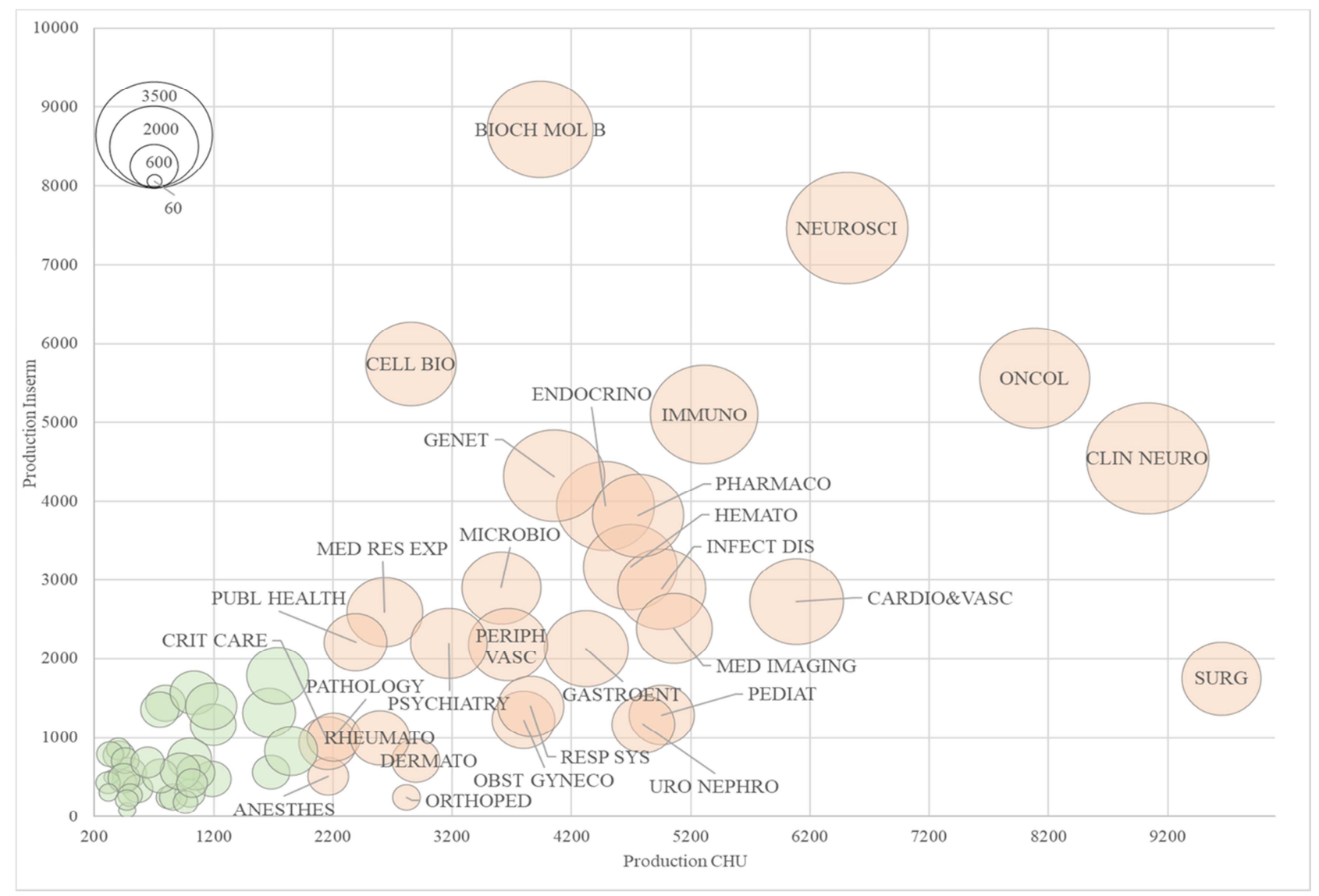

\section{FIGURE 4}

Vue d'ensemble des disciplines des publications issues de la collaboration CHU-Inserm.

Liste abréviation disciplines affichées (partie orange):

ANESTHES = Anesthesiology $;$ BIOCH MOL B = Biochemistry \& molecular biology ; BUS FIN = Business, finance $;$ CARDIO\&VASC = Cardiac \& cardiovascular systems ; CELL BIO = Cell biology ; CLIN NEURO = Clinical neurology ; CRIT CARE = Critical care medicine ; DERMATO = Dermatology ; ENDOCRINO = Endocrinology \& metabolism ; ENG MARINE = Engineering, marine ; ENG OC = Engineering, ocean ; GASTROENT = Gastroenterology \& hepatology ; GENET = Genetics \& heredity ; HEMATO = Hematology ; HORTICUL = Horticulture ; IMMUNO = Immunology ; INFECT DIS = Infectious diseases ; LIMNO = Limnology ; MED RES EXP = Medicine, research \& experimental ; MICROBIO = Microbiology ; NEUROSCI = Neurosciences ; OBST GYNECO = Obstetrics \& gynecology ; ONCOL = Oncology ; ORTHOPED = Orthopedics ; PATHOLOGY = Pathology ; PEDIAT = Pediatrics ; PERIPH VASC = Peripheral vascular disease ; PHARMACO = Pharmacology \& pharmacy ; PSYCHIATRY = Psychiatry $;$ PUBLIC ADM = Public administration ; PUBL HEALTH = Public, environmental \& occupational health ; MED IMAGING = Radiology, nuclear medicine \& medical imaging ; RESP SYS = Respiratory system ; RHEUMATO = Rheumatology . 


\begin{tabular}{l|c|c|c|c|c|c|} 
& \multicolumn{5}{|c|}{ Toutes disciplines confondues } \\
& Nb d'articles & \% France & \% UE28 & Nb d'articles & \% France & \% UE28 \\
\hline CEA & 20390 & 6.8 & 1 & 24264 & 6.9 & 1 \\
CHU & 52069 & 17.4 & 2.6 & 60653 & 17.3 & 2.4 \\
CNRS & 125028 & 41.8 & 6.1 & 150237 & 42.8 & 6 \\
\hline INRA & 15819 & 5.3 & 0.8 & 19154 & 5.5 & 0.8 \\
\hline Inria & 4583 & 1.5 & 0.2 & 6978 & 2 & 0.3 \\
\hline Inserm & 31824 & 10.6 & 1.6 & 42947 & 12.2 & 1.7 \\
\hline Institut Pasteur (Paris, Lille) & 4907 & 1.6 & 0.2 & 5270 & 1.5 & 0.2 \\
\hline IRD-France & 8895 & 3 & 0.4 & 13456 & 3.8 & 0.5 \\
\hline UNICANCER* & 7153 & 2.4 & 0.4 & 9993 & 2.8 & 0.4 \\
\hline universités** & 192776 & 64.4 & 9.5 & 236954 & 67.4 & 9.5 \\
\hline France & 299383 & - & 14.7 & 351392 & - & 14 \\
\hline UE28 & 2033769 & - & - & 2504470 & - & - \\
\hline
\end{tabular}

\section{TABLEAU I}

Nombre de publications et parts nationale et européenne (\%) des membres d'AVIESAN, toutes disciplines confondues, 2006-2015.

Données : Clarivate Analytics; Traitement : CNCR

* UNICANCER est membre associé d'AVIESAN.

** L'entité « universités » regroupe toutes les universités identifiées dans InCites.

Attention : calculés en compte de présence, les chiffres des établissements ne sont pas additionnables. 


\begin{tabular}{|c|c|c|c|}
\hline & \multicolumn{3}{|c|}{ Toutes disciplines confondues } \\
\hline & $\begin{array}{c}\text { ICN } \\
2006-2010 \\
\%(\mathrm{~N})\end{array}$ & $\begin{array}{c}\text { ICN } \\
2011-2015 \\
\%(\mathrm{~N})\end{array}$ & Evolution \\
\hline CEA & $1,53(20390)$ & $1,76(24264)$ & ن \\
\hline CHU & $1,24(52069)$ & $1,55(60653)$ & ن \\
\hline CNRS & $1,3(125028)$ & $1,36(150237)$ & 仓 \\
\hline INRA & $1,44(15819)$ & $1,46(19154)$ & 仓 \\
\hline Inria & $1,18(4583)$ & $1,1(6978)$ & Z \\
\hline Inserm & $1,71(31824)$ & $1,8(42947)$ & 仓 \\
\hline Institut Pasteur (Paris, Lille) & $1,93(4907)$ & $2,13(5270)$ & 仓 \\
\hline IRD-France & $1,44(8895)$ & $1,35(13456)$ & 7 \\
\hline UNICANCER* & $1,92(7153)$ & $2,33(9993)$ & 仓 \\
\hline Universités** & $1,21(192776)$ & $1,31(236954)$ & 仓 \\
\hline France & $1,18(299383)$ & $1,28(351392)$ & ن \\
\hline UE28 & $1,08(2033769)$ & $1,11(2504470)$ & 仓 \\
\hline
\end{tabular}

\section{TABLEAU II}

Indice d'impact normalisé et nombre d'articles (entre parenthèses) des membres d'AVIESAN, toutes disciplines confondues : évolution entre 2006-2010 et 2011-2015. ICN : Indice de Citation Normalisé.

Données : Clarivate Analytics; Traitement : CNCR

* UNICANCER est membre associé d'AVIESAN.

** L'entité « universités » regroupe toutes les universités identifiées dans InCites. Attention : calculés en compte de présence, les chiffres des établissements ne sont pas additionnables. 


\begin{tabular}{|c|c|c|c|c|}
\hline & \multicolumn{4}{|c|}{ Toutes disciplines confondues } \\
\hline & \multicolumn{2}{|c|}{$\begin{array}{c}\text { Part de production } \\
\text { 2006-2010 } \\
\%(\mathrm{~N})\end{array}$} & \multicolumn{2}{|c|}{$\begin{array}{c}\text { Part de production } \\
\text { 2011-2015 } \\
\%(\mathrm{~N})\end{array}$} \\
\hline & En Anglais & En Français & En Anglais & En Français \\
\hline CEA & $98,4(20151)$ & $1,4(281)$ & $99(24158)$ & $0,8(184)$ \\
\hline CHU & 75 (39165) & 24,9 (12974) & $84,1(51185)$ & $15,7(9579)$ \\
\hline CNRS & 97,2 (120559) & $2,7(3365)$ & $98(146734)$ & $1,9(2814)$ \\
\hline INRA & $95,8(15152)$ & $4(634)$ & $97,2(18615)$ & $2,7(521)$ \\
\hline Inria & $98,5(4536)$ & $1,5(68)$ & $99,2(6932)$ & $0,8(57)$ \\
\hline Inserm & $93,2(29647)$ & $6,8(2154)$ & $95(40825)$ & 4,9 (2119) \\
\hline Institut Pasteur (Paris, Lille) & $97,3(4777)$ & $2,7(131)$ & $97,9(5169)$ & $2,1(109)$ \\
\hline IRD-France & $96,6(8950)$ & $3,1(283)$ & $97,7(13498)$ & $2,1(288)$ \\
\hline UNICANCER* & $84,3(6031)$ & $15,6(1116)$ & $88,5(8830)$ & $11,4(1138)$ \\
\hline Universités** & $91,9(176532)$ & $7,7(14861)$ & $94,2(222189)$ & $5,5(12983)$ \\
\hline
\end{tabular}

\section{TABLEAU III}

Evolution des parts de production (en \%) et du nombre de publications correspondantes (entre parenthèses) en anglais et en français des membres d'AVIESAN, 2006-2015.

* UNICANCER est membre associé d'AVIESAN.

** L'entité « universités » regroupe toutes les universités identifiées dans InCites.

Attention : calculés en compte de présence, les chiffres des établissements ne sont pas additionnables. 


\begin{tabular}{|l|c|c|} 
& $\begin{array}{c}\text { Collaboration } \\
\text { Internationale } \\
\mathbf{2 0 0 6 - 2 0 1 0} \\
\%(\mathrm{~N})\end{array}$ & $\begin{array}{c}\text { Collaboration } \\
\text { Internationale } \\
\mathbf{2 0 1 1 - 2 0 1 5} \\
\%(\mathrm{~N})\end{array}$ \\
\hline CEA & $55,2(11255)$ & $60,3(14631)$ \\
\hline CHU & $29,4(15308)$ & $37,9(22987)$ \\
\hline CNRS & $52(65015)$ & $57,7(86687)$ \\
\hline INRA & $45(7119)$ & $49,6(9500)$ \\
\hline Inria & $45,9(2104)$ & $53,6(3740)$ \\
\hline Inserm & $44,4(14130)$ & $49,8(21388)$ \\
\hline Institut Pasteur(Paris, Lille) & $57,9(2841)$ & $60(3162)$ \\
\hline IRD-France & $67,1(5969)$ & $69,7(9379)$ \\
\hline UNICANCER* & $39(2790)$ & $45(4497)$ \\
\hline Universités** & $47(90605)$ & $53,3(126296)$ \\
\hline France & $48,7(145800)$ & $55,2(193968)$ \\
\hline UE28 & $35,9(730123)$ & $40,8(1021824)$ \\
\hline
\end{tabular}

\section{TABLEAU IV}

Parts en \% et nombre d'articles en collaboration internationale (entre parenthèses) dans la production des membres d'AVIESAN, toutes disciplines confondues : évolution entre 2006-2010 et 2011-2015

Données : Clarivate Analytics; Traitement : CNCR

* UNICANCER est membre associé d'AVIESAN.

** L'entité « universités » regroupe toutes les universités identifiées dans InCites.

Attention : calculés en compte de présence, les chiffres des établissements ne sont pas additionnables. 


\begin{tabular}{|c|c|c|c|c|c|c|c|c|c|}
\hline $\begin{array}{c}\text { Collaboration CHU \& Organismes } \\
\text { TOP } 10 \text { Disciplines }\end{array}$ & 空 & 艺 & $\underset{\underline{z}}{\overleftrightarrow{z}}$ & $\stackrel{\underline{a}}{\underline{\Xi}}$ & 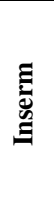 & 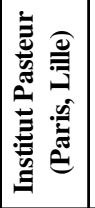 & ๙ิ & 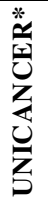 & 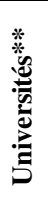 \\
\hline CLINICAL NEUROLOGY & 2 & 3 & & 3 & 1 & & & & 1 \\
\hline NEUROSCIENCES & 1 & 1 & & 2 & 2 & & & & 4 \\
\hline BIOCHEMISTRY MOLECULAR BIOLOGY & 3 & 2 & 2 & & 5 & 7 & 10 & 7 & \\
\hline IMMUNOLOGY & 7 & 8 & 8 & & 4 & 3 & 3 & 10 & 5 \\
\hline MULTIDISCIPLINARY SCIENCES & 5 & 4 & 4 & & 9 & 4 & 4 & & \\
\hline GENETICS HEREDITY & 4 & 5 & 6 & & 6 & 6 & & 6 & \\
\hline ONCOLOGY & 10 & 7 & & & 3 & & & 1 & 2 \\
\hline RADIOLOGY NUCLEAR MEDICINE MEDICAL IMAGING & 6 & 9 & & 1 & & 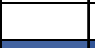 & & 3 & 9 \\
\hline INFECTIOUS DISEASES & & & 9 & & & 2 & 1 & & 6 \\
\hline MICROBIOLOGY & & 10 & 5 & & & 1 & 2 & & \\
\hline CELL BIOLOGY & 9 & 6 & 7 & & & 9 & & 4 & \\
\hline SURGERY & & & & & & & & 5 & 3 \\
\hline ENDOCRINOLOGY METABOLISM & & & 3 & & 7 & 10 & & & \\
\hline HEMATOLOGY & & & & & 8 & & & 2 & 10 \\
\hline PHARMACOLOGY PHARMACY & & & & & 10 & 8 & 9 & 8 & 8 \\
\hline VIROLOGY & & & & & & 5 & 6 & & \\
\hline NUTRITION DIETETICS & & & 1 & & & & & & \\
\hline ENGINEERING BIOMEDICAL & & & & 4 & & & & & \\
\hline NEUROIMAGING & & & & 5 & & & & & \\
\hline PUBLIC ENVIRONMENTAL OCCUPATIONAL HEALTH & & & & & & & 5 & & \\
\hline CARDIAC CARDIOVASCULAR SYSTEMS & & & & 10 & & & & & 7 \\
\hline COMPUTER SCIENCE INTERDISCIPLINARY APPLICATIONS & & & & 6 & & & & & \\
\hline TROPICAL MEDICINE & & & & & & & 7 & & \\
\hline MATHEMATICAL COMPUTATIONAL BIOLOGY & & & & 7 & & & & & \\
\hline PSYCHIATRY & 8 & & & & & & & & \\
\hline PARASITOLOGY & & & & & & & 8 & & \\
\hline COMPUTER SCIENCE ARTIFICIAL INTELLIGENCE & & & & 8 & & & & & \\
\hline PATHOLOGY & & & & & & & & 9 & \\
\hline ENGINEERING ELECTRICAL ELECTRONIC & & & & 9 & & & & & \\
\hline FOOD SCIENCE TECHNOLOGY & & & 10 & & & & & & \\
\hline
\end{tabular}

\section{TABLEAU V}

Dix premières (par volume) disciplines (catégories WoS) de co-publication des CHU avec les membres d'AVIESAN, 2006-2015.

Données : Clarivate Analytics; Traitement : CNCR

* UNICANCER est membre associé d'AVIESAN.

** L'entité « universités » regroupe toutes les universités identifiées dans InCites. 